\title{
L'utilisation des données empiriques dans le cadre du contrôle des discriminations par le juge administratif
}

\author{
Caroline Lantero, Maître de conférences en droit public, UCA EA4232 \\ et \\ François Cafarelli, Maître de conférences en droit public, Université de la Réunion, CRJ \\ EA14
}

Revue française d'administration publique, ENA, 2020, vol. I (n¹73), pp. 43-54

Chargé d'identifier et de sanctionner des pratiques discriminatoires, le juge administratif est placé dans une situation inconfortable dès lors qu'il lui faut articuler les situations particulières des justiciables avec les outils empiriques plus généraux et objectifs permettant de mettre en évidence des discriminations. Cette dialectique de la lutte contre les discriminations conduit le juge à ne pas nier la nécessité de faire appel aux données empiriques pour résoudre les litiges même s'il fait preuve d'une infinie discrétion dans leur mise en œuvre.

Mots-clefs

discriminations, juge administratif,statistiques

\begin{abstract}
When in charge of identifying and censuring discrimination, administrative courts are placed in an uncomfortable position since they have to articulate individual cases with more general and empirical tools to highlight discriminations. The fight against discrimination leads the judges to accept the need for such tools when disputes have to be resolved, but they also show an infinite discretion in their implementation.
\end{abstract}

Keywords

Discrimination, judge, statistics

En dépit de l'importance de la thématique discriminatoire et du nombre tout aussi important de travaux scientifiques essayant de l'appréhender sur le terrain juridique, l'approche contentieuse des phénomènes discriminatoires demeure difficile. Faute de définitions partagées, faute de catégories clairement délimitées, faute d'outils de mesure standardisés, le défi probatoire est encore immense pour le justiciable qui doit faire face à quatre difficultés.

Premièrement, il existe encore une difficulté à penser la discrimination dans un système juridique structuré autour du principe d'égalité. Pour s'en convaincre, il suffit d'évoquer la confusion, parfaitement synthétisée par Jean-Pierre Marguenaud préfaçant la thèse de Delphine Tharaud (Tharaud, 2013), entre les principes d'égalité et de nondiscrimination et la possibilité de considérer que des différenciations ne portent pas atteinte à ce principe d'égalité. Cette difficulté, ancienne, a donné lieu à des débats, parfois violents, sur la portée du principe d'égalité (Pellissier, 1996, 1), puis à des débats, encore plus violents, sur la notion de discrimination, que le droit français a répugné à définir. Le terme « discrimination », dont l'étymologie renvoie à l'action - neutre - de distinguer, intègre en effet tardivement le droit positif et sous la forme d'une expression à connotation négative dans la loi Pleven de 1972 relative à la lutte contre le racisme ${ }^{1}$, mais ne sera pas défini. Pas

\footnotetext{
${ }^{1}$ Loi $\mathrm{n}^{\circ} 72-546$ du 1 juillet 1972 relative à la lutte contre le racisme.
} 
davantage que dans les multiples lois de ratification de traités internationaux, lesquelles n'étaient pas assorties de portée pratique ${ }^{2}$. Pas davantage que dans les jurisprudences fort connues du Conseil constitutionnel, comme du Conseil d'État, effleurant la notion au prisme du seul principe d'égalité, tantôt en assimilant égalité et non-discrimination ${ }^{3}$, tantôt en jugeant que la différenciation (et non pas d'ailleurs la discrimination) n'est pas toujours contraire au principe d'égalité ${ }^{4}$, tout en précisant que ce dernier n'ouvre pas pour autant droit à la différence ${ }^{5}$, souvent en se bornant à limiter la discrimination à une atteinte au principe d'égalité. Comment dès lors, résoudre les difficultés conceptuelles posées par les notions de discrimination positive (Tharaud, 2013) ou compensatoire (Cafarelli, 2007), qui sont venues frontalement heurter le principe d'égalité ?

En outre, le droit européen ${ }^{6}$ et le droit de l'Union $^{7}$ ont fortement influencé le droit français et, pourrait-on dire, l'ont forcé en dehors de sa zone de confort que constituait le seul principe d'égalité, pour qu'il évolue vers un véritable droit de la non-discrimination, plus opératoire, conduisant Gwennaelle Calvès à identifier une «technique de qualification des ruptures d'égalité » (Calvès, 2012, 9).

Deuxièmement, le tabou lié à l'absence de normes a laissé la place à la cacophonie de la profusion de normes notamment avec la loi du 28 mai 2008 portant diverses dispositions d'adaptation au droit communautaire dans le domaine de la lutte contre les discriminations ou une modification de la Constitution pour favoriser l'égalité des femmes et des hommes ${ }^{8}$. Désormais, il est également question d'égalité réelle, différente de l'égalité formelle instituée en vertu du principe d'égalité ${ }^{9}$. Ces textes n'ont pas pleinement contribué à fixer les contours de la notion de discrimination, mais ils ont eu pour conséquence de resserrer la notion de discrimination autour de son acception négative. La définition ici retenue est donc celle de l'énumération presque exhaustive proposée par l'article 225-1 du code pénal pour mesurer l'étendue des branches de discrimination à mesurer.

Troisièmement, l'interdiction des discriminations a été logiquement assortie de la faculté de s'en prévaloir devant un juge, mais ce dernier n'est pas toujours très à l'aise ni bien armé pour en connaître. Il n'est ici question que du seul juge administratif dont l'office est bien particulier puisqu'il statue sur des décisions et non des comportements, et dont la démarche probatoire est singulière par rapport à celle des autres juges puisqu'il compose en permanence avec les limites de la preuve (Liéber et Botteghi, 2009). Le juge administratif souffre de difficultés (de réticences ?) à manier une approche catégorielle, ce qui donne à son contrôle une portée individualiste et casuistique à l'origine de solutions particulièrement rétives à la prise en compte de groupes sociaux. Il y a donc une différence d'approche en fonction des catégories concernées. Certaines sont plus facilement identifiables que d'autres

\footnotetext{
${ }^{2}$ Loi n 71-392 du 28 mai 1971 autorisant l'adhésion à la convention internationale sur l'élimination de toutes les formes de discrimination raciale, ouverte à la signature le 07-03-1966; Loi n 81-257 du 15 avril 1981 autorisant l'approbation de la convention internationale du travail $\mathrm{n}^{\circ} 111$ concernant la discrimination en matière d'emploi et de profession ; Loi n ${ }^{\circ} 83-561$ du 1er juillet 1983 autorisant la ratification d'une convention sur l'élimination de toutes les formes de discrimination à l'égard des femmes, ouverte à la signature à New-York le 01-03-1980.

${ }^{3}$ CC, 18 janvier 1985 , déc. $n^{\circ} 84182$ DC.

${ }^{4}$ CE, Sect., 13 mai 1994, Commune de Dreux, Rec. 233 ; CE, Sect., 29 décembre 1997, Commune de Gennevilliers, Rec. 499.

${ }^{5}$ CE, Ass., 28 mars 1997, Sté Baxter, n 179049.

${ }^{6}$ Art. 14 de la $\mathrm{C}^{\circ} \mathrm{EDH}$.

${ }^{7}$ Directive 2000/43 du 29 juin 2000 relative à l'égalité de traitement entre les personnes sans distinction de race ou d'origine ethnique ; directive 2000/78 du 27 novembre 2000 sur l'interdiction des discriminations en matière d'accès au travail et à l'emploi ; directive n²006/54/CE du Parlement européen et du Conseil, du 5 juillet 2006, relative à la mise en œuvre du principe de l'égalité des chances et de l'égalité de traitement entre hommes et femmes en matière d'emploi et de travail ; mais aussi art. 19 TFUE.

${ }^{8}$ Loi constitutionnelle ${ }^{\circ} 2008-724$ du 23 juillet 2008 de modernisation des institutions de la Ve République.

${ }^{9}$ Loi n ${ }^{\circ} 2014-873$ du 4 août 2014 pour l'égalité réelle entre les femmes et les hommes.
} 
en ce qu'elles reposent sur des données acceptées comme objectives : l'âge, le genre, le handicap, la nationalité. Pour les autres catégories (ethnie, race, religion, orientation sexuelle, origine), la Constitution prohibe toujours toute distinction. Ce refus historique des distinctions fondées sur ces catégories entraîne paradoxalement leur inexistence juridique (Lantero, 2009). On les désigne peu. On les mesure encore moins.

Pourtant, le juge administratif a lui aussi été contraint de manipuler des catégories, en matière de discriminations directes comme indirectes. Et il doit de plus composer avec une administration qui se garde bien d'édicter des mesures directement discriminatoires à l'endroit de groupes sociaux nommés et visés : les femmes musulmanes n'étaient évidemment pas explicitement visées dans les arrêtés antiburkini, ni les personnes de confession musulmane ou juive dans l'affaire de la "soupe aux cochons », ni les Roms dans les arrêtés d'interdiction de fouille des poubelles, etc. ${ }^{10}$ La question des groupes discriminés est, en ce domaine, la plus intéressante, car on perçoit à la fois l'appétence du juge à disposer d'une grille de compréhension des catégories discriminées, et les efforts finalement fournis pour faire entrer le problème des groupes discriminés dans le schéma classique du principe d'égalité, voire, dans la seule question du contrôle de proportionnalité de la mesure.

Quatrièmement, et sans doute principalement, le système normatif français résiste encore à une approche catégorielle et le juge - pourtant tenu de statuer - ne dispose pas d'outils de mesure de la discrimination. Tout le débat juridique se résume alors ainsi : dans un contentieux donné, la discrimination est seulement une question d'espèce posant des difficultés probatoires, que le juge peut, le cas échéant, résoudre en allant chercher dans les données empiriques, celles qui sont le produit de l'expérience. Évidemment, toutes les données empiriques ne se valent pas. Il est difficile de mettre sur le même plan l'expérience isolée et située d'un justiciable et des études statistiques réalisées dans le cadre d'une méthodologie objective. Dès lors, par «données empiriques », on entend la connaissance acquise, soit par l'observation, soit par l'expérimentation, et revêtue d'une certaine autorité intellectuelle et d'une certaine force probatoire.

Cette définition des «données empiriques » porte en elle la contradiction, que le juge devra résoudre, entre les éléments de preuve rapportés par le justiciable, parfois empreints de subjectivité, et les éléments de preuve de l'existence d'une discrimination systémique, davantage marqués par l'objectivité. Elle invite ainsi le chercheur à cerner la place de ces données empiriques dans le contentieux des discriminations, idéalement en le conduisant à identifier des éléments permettant de mieux comprendre la démarche du juge, ses exigences et la mécanique de ses raisonnements face au phénomène discriminatoire.

Une telle recherche suscite inévitablement la frustration. En évitant l'écueil du regard militant souvent présent en matière de lutte contre les discriminations, elle met en évidence l'étendue de la complexité du travail juridictionnel et les limites de l'analyse doctrinale juridique qui ne peut pas aller plus loin que le seuil du for intérieur du magistrat. En d'autres termes, cette contribution, disons-le d'emblée, se conclut par un troublant constat d'échec. Troublant en ce qu'il ne marque pas l'arrivée définitive au bout d'une voie sans issue mais dit plutôt l'ampleur de ce que nous ignorons et invite à poursuivre l'étude sur le temps long. Dans la mesure où la prise en compte des données empiriques par le juge ne fait guère de doute tant elle est nécessaire dans le cadre du contrôle des discriminations (I), il y a en effet tout lieu de penser que leur utilisation par le juge, aujourd'hui rare, discrète, et rétive à l'analyse (II) laissera la place, demain, à un usage beaucoup plus assumé.

\section{Un recours réfléchi aux données empiriques}

\footnotetext{
${ }^{10}$ Notamment CE, 5e et 4e Ch.R, 15 novembre 2017, LDH, n 403275 ; CE, Ord., 26 août 2016, Ligue des droits autres et Association de défense des droits de l'homme Collectif contre l'islamophobie en France, $\mathrm{n}^{\circ} 403578$.
} 
Face aux difficultés rencontrées par les justiciables dans la démonstration de l'existence d'une discrimination, le juge administratif a opportunément aménagé (et non renversé) la charge de la preuve en instaurant une présomption. Ce mécanisme fort utile apparaît néanmoins insuffisant, si bien que le juge est amené à le compléter par la prise en compte de données empiriques établies par le requérant (A) ou par des sachants (B).

\section{A. Une présomption souvent insuffisante}

Dans le procès administratif, le laboratoire du maniement des catégories discriminées est incontestablement le contentieux de la fonction publique. Dans ce domaine, l'absence d'outil de mesure et de grille de lecture de la discrimination a posé de sérieuses difficultés aux administrés, confronté à un obstacle majeur : le régime objectif de la preuve qui prévaut en contentieux administratif.

En avril 2009, peu avant la retentissante décision Perreux, le Conseil d'État ${ }^{11}$ devait rechercher l'existence d'une discrimination raciale dans le déroulé d'un concours et, comme dans la décision Barrel de $1954^{12}$, son travail a été grandement facilité par l'absence de contestation sérieuse de l'administration aux éléments concordants et affligeants apportés par le requérant (Calvès, 2009). Toutefois, le Rapporteur public Jean-Philippe Thiellay avait souligné à cette occasion les difficultés probatoires posées par le contentieux de la discrimination, ce que l'on apprend sous la plume de Mathias Guyomar dans ses conclusions sur la décision Perreux : «nous avions constaté les limites des outils de contrôle dont dispose le juge de l'excès de pouvoir lorsqu'il est soutenu devant lui qu'une décision administrative est empreinte de discrimination, sauf à ce que l'élément qui révèle l'existence de la discrimination alléguée ressorte clairement des pièces du dossier » (2009).

Le Vice-président du Conseil d'État le rappelait à la fin de l'année 2018 : « Mal adapté aux cas de discrimination dès lors que les requérants peinent souvent à réunir des preuves suffisantes de la discrimination dont ils disent avoir été victimes » (Lasserre, 2018), ce régime a été aménagé pour le contentieux de la discrimination. En 2009, le Conseil d'État accepte dans la décision Perreux ${ }^{13}$ que la personne s'estimant victime d'une discrimination se contente d'amorcer une argumentation au soutien de son moyen et qu'il incombe au défendeur « de produire tous ceux permettant d'établir que la décision attaquée repose sur des éléments objectifs étrangers à toute discrimination ». Il ne s'agit pas tout à fait d'un renversement de la charge de la preuve puisque le défendeur ne doit pas prouver le contraire, mais doit prouver autre chose. Le Conseil d'État précise également que le juge doit former sa conviction de manière minutieuse, dans le plus grand respect du contradictoire et, s'il le faut, en ordonnant une mesure d'instruction. La discrimination ne fut pas reconnue en l'espèce, mais l'a été peu après, dans une affaire similaire ${ }^{14}$ et la méthodologie probatoire issue de la décision Perreux a permis d'établir l'existence d'un certain nombre de pratiques discriminatoires, mais pas autant que l'on pouvait peut-être s'y attendre (Dumortier, 2017). Sans énumérer tous les litiges dans lesquels l'administration parvient à démontrer que la décision repose sur des éléments objectifs étrangers à toute discrimination, il est encore fréquent que le requérant ne franchisse pas la première étape et «n'apporte aucun élément permettant de faire présumer » une mesure discriminatoire ${ }^{15}$. Et c'est éventuellement à ce stade qu'interviennent les données empiriques comme aide à la preuve.

\footnotetext{
${ }^{11} \mathrm{CE}, 5 \mathrm{e}$ et $4 \mathrm{e}$ SSR, 10 avril 2009, El Haddioui, $\mathrm{n}^{\circ} 311888$.

${ }^{12}$ CE, Ass., 28 mai 1954, Barel, ${ }^{\circ} 28238$.

${ }^{13}$ CE, Ass., 30 octobre 2009, Perreux, $\mathrm{n}^{\circ} 298348$.

${ }^{14} \mathrm{CE}, 6$ e et 1e SSR, 10 janvier 2011 , Mme Lévèque, $\mathrm{n}^{\circ} 325268$.

${ }^{15}$ CE 23 juin 2010, n 328953 ; CE 27 juillet 2016, n 393669 ; CE 24 mars 2014, n 363902.
} 
Ces données émanent essentiellement de sachants extérieurs, mais le requérant peut tout à fait les produire lui-même. Par exemple, le juge accepte de prendre en compte les «tests de comparaison» dans lesquels le requérant tente d'établir le «traitement plus favorable ${ }^{16}$ et non justifié des collègues, mais leurs résultats sont rarement retenus comme suffisants par eux-mêmes pour établir une présomption (Mercat-Bruns et autres, 2017). Ainsi n'est-il pas possible d'évoquer l'avènement de « la preuve par la statistique » en matière de discrimination (Roussel et Nicolas, 2017), notamment en raison de l'interdiction récurrente des statistiques ethniques, et il faut sans doute préférer l'intitulé mesuré choisi par SophieJustine Lieber : «De l'usage des statistiques dans la présomption des discriminations : oui ; mais... » (Lieber, 2018). Dans cette nouvelle affaire de recrutement, un candidat malheureux à un concours avait tenté d'établir une discrimination entre les candidats, fondés d'une part sur l'âge, d'autre part sur leur localisation géographique. Le Conseil d'État écarte le calcul de probabilité relatif à l'âge des candidats, et estime que les statistiques proposées quant au critère géographique "ne sont [pas] de nature à faire présumer une atteinte au principe d'égalité de traitement ». Corrélation, même avérée, n'est pas causalité. En revanche et c'est toute la portée de la décision, il accepte - pour identifier des éventuels critères de discrimination implicite - de prendre en considération ces données statistiques à condition, ce qui n'était pas le cas, qu'elles soient fiables et significatives (Lieber, 2018).

\section{B. Des savoirs extérieurs toujours choisis}

Dans le contentieux de la discrimination, le juge administratif apparaît très sélectif quant aux expertises extérieures. Une recherche exhaustive n'a ainsi pas permis d'identifier la moindre référence, dans une décision ou dans des conclusions de Rapporteur public, aux travaux de l'Observatoire des inégalités, de l'Observatoire des discriminations ou de la doctrine. Concernant l'écoute des sachants, le juge administratif semble mieux considérer ceux qu'il invite (l'expert judiciaire, ou l'amicus curiae), ou ceux que la loi habilite à se faire entendre (la HALDE, puis le Défenseur des droits) ce qui est plus fréquent, mais moins propice à l'analyse quant aux rapports d'influence ou enfin, mais leur influence est encore moins palpable, les sachants « institués ».

L'expert désigné par le juge ${ }^{17}$ est probablement le sachant invité le plus indiscutable puisque la désignation émane du juge lui-même. Pour autant, aucune jurisprudence n'existe s'agissant de mesurer une discrimination, ce qui n'est pas étonnant compte tenu de ce que le juge administratif serait bien en peine d'en rédiger la mission. Quoique, pour des litiges qui ne concerneraient pas des questions d'origine ethnique ou de race, un expert statisticien pourrait être missionné afin d'établir des données chiffrées fiables et significatives utiles à la présomption d'une discrimination. L'amicus curiae est aussi invité par le juge ${ }^{18}$ à donner son avis au regard d'une compétence ou d'une connaissance qui «seraient de nature à l'éclairer utilement sur la solution à donner à un litige », y compris sur une question de droit. Une seule illustration contentieuse a émergé en matière de discrimination, dans la très déstabilisante affaire du retrait des menus de substitution des cantines de Chalon-sur-Saône (Roman, 2017). Le tribunal administratif de Dijon ${ }^{19}$ a sollicité la tierce intervention des amicii curiae pour finalement dire qu'il ne se servirait pas de leur avis, tout en s'en servant...et mal. Le tribunal avait en effet sollicité, d'une part, la Commission nationale consultative des droits de l'homme qui avait souligné que la délibération partait d'une interprétation erronée des principes de laïcité et d'égalité ainsi que d'une méconnaissance de l'intérêt supérieur de

\footnotetext{
${ }^{16}$ CAA Bordeaux, 10 mars 2014, $\mathrm{n}^{\circ}$ 13BX00711.

${ }^{17}$ Art. R. 532-1 ou R. 621-1 CJA.

18 Art. R. 625-3 CJA.

${ }^{19}$ TA Dijon, 28 août 2017, n $1502100,1502726$.
} 
l'enfant, et, d'autre part, le Défenseur des droits qui avait estimé qu'au regard du contexte de son adoption et de l'absence de contrainte de service, la délibération attaquée " pourrait être susceptible de revêtir un caractère discriminatoire » ». Ne souhaitant probablement pas ouvrir cette dernière porte, le tribunal a jugé, tout en précisant qu'il n'était pas «besoin de tenir compte des observations dépourvues du caractère général requis par l'article R. 625-3 du code de justice administrative », que la mesure attaquée portait atteinte à l'intérêt supérieur de l'enfant. C'est sur ce point que le jugement a été annulé, car personne n'avait soulevé ce moyen. La CAA de Lyon a tout de même annulé la délibération de manière plus « courageuse » en n'ignorant pas l'éléphant dans la pièce : l'absence de contradiction entre les principes de laïcité et de neutralité et les menus de substitution ${ }^{20}$.

Bien qu'aucun mystère n'existât sur le fait que l'amicus curiae n'était ni expert judiciaire, ni témoin (Melleray, 2014), les espoirs reposant alors sur cette innovation sont probablement aujourd'hui déçus, surtout en matière de lutte contre les discriminations. En effet, le juge administratif a tôt fait de refuser ce statut aux avis spontanés. Dans l'affaire GISTI et FAMPIL ${ }^{21}$, le Centre de recherche et d'étude sur les droits fondamentaux (CREDOF) de l'Université Paris-Est Nanterre La Défense avait soumis une étude sur la justiciabilité des droits sociaux en se présentant comme amicus curiae, mais cette étude n'a pas été visée. Gaëlle Dumortier indiquait en ce sens à sa formation dans ses conclusions «qu'une personne vous demande à être votre amie ne vous interdit pas plus qu'elle ne vous oblige à l'inviter, ni même à lui répondre explicitement » (Dumortier, 2012). Quant à ceux qui sont dûment invités, le Conseil d'État a précisé qu'ils devaient se borner à produire des observations générales, conformément à la lettre de l'article R 623-5 du code de justice administrative et qu'à défaut, le juge devait prendre en compte les seules observations d'ordre général, juridiques ou factuelles ${ }^{22}$.

La HALDE puis le Défenseur des droits sont des sachants habilités. Dans cette même décision du 11 avril 2012, était scellé le statut procédural de la (HALDE), instituée par la loi du 30 décembre 2004 lui reconnaissant le droit d'être entendue par les juridictions. Quelques semaines plus tôt, le Conseil d'État avait précisé que ce droit d'être entendue par les juridictions administratives «ne lui conférait pas la qualité d'intervenante dans un litige de plein contentieux ${ }^{23}$. Dans les résumés de la décision GISTI et FAMPIL du 11 avril 2012, il est précisé que «ses productions sont visées sans être analysées et il n'est pas répondu à ses moyens propres dans les motifs de la décision ». Selon la même logique, le Défenseur des droits, institué par la loi organique $\mathrm{n}^{\circ}$ 2011-333 du 29 mars 2011, est maintenu en statut d'observateur lorsqu'il intervient de lui-même. Le juge l'écoute, mais ne lui répond pas.

Viennent enfin les références aux travaux d'experts «institués » tels que l'Académie française, personne morale de droit public à statut particulier, le comité consultatif national d'éthique, autorité administrative indépendante, la CNCDH créée en 1947 et «assimilée » à une autorité administrative indépendante depuis 2007 et le Défenseur des droits, autorité constitutionnelle indépendante. Mais leur utilisation - à tout le moins par le Conseil d'État n'est transparente que lorsque les conclusions des Rapporteurs publics sont accessibles. Car elle n'apparaît pas dans les décisions. En somme, les experts doivent être dans la lumière, mais leurs travaux ne sont utilisés que dans l'ombre de l'instruction.

\section{Un recours feutré aux données empiriques}

\footnotetext{
${ }^{20}$ CAA Lyon, 23 octobre 2018, Commune de Chalon-Sur-Saône, n 17 LY03323 et 17LY03328.

${ }^{21}$ CE, Ass., 11 avril 2012, $n^{\circ} 322326$.

${ }^{22} \mathrm{CE}, 8 \mathrm{e}$ et $3 \mathrm{e}$ SSR, 6 mai $2015, \mathrm{n}^{\circ} 375036$.

${ }^{23} \mathrm{CE}, 7 \mathrm{e}$ et 2e SSR, 22 février 2012, Chambre Régionale d'Agriculture de PACA, n $343410,343438$.
} 
Extrêmement discret dans les décisions (A), le recours aux savoirs extérieurs est encore plus confidentiel et encore moins sujet à l'analyse lorsqu'on le cherche autour de la décision, et plus précisément, dans les conclusions du Rapporteur public (B).

\section{A. Des décisions laconiques}

Le Défenseur des droits s'invite régulièrement dans les procès, soit totalement spontanément, soit parce que la procédure lui a été « communiquée pour observations » par la juridiction, sans actionner pour autant le mécanisme de l'amicus curiae. Or, il est rigoureusement impossible, à la lecture des décisions de justice, de mesurer l'influence des données ou observations communiquées. En pratique certes, les décisions du Défenseur des droits tendant à présenter des observations devant une instance sont publiées sur son site internet, avec lesdites observations. Indépendamment des questionnements relatifs au caractère public - fussent-elles anonymisées - des observations, ce qui n'a pas encore fait l'objet d'un contentieux, mais peut légitimement être regardé comme faisant grief, cette publication est intéressante pour l'analyse de l'influence des observations sur la solution du juge. Intéressante, mais pas décisive. Compte tenu de l'absence de référence aux observations dans les décisions de justice, à tout le moins devant le Conseil d'État, la corrélation n'emporte pas davantage ici causalité.

Pour illustration, dans une décision du 26 janvier 2015, le Conseil d'État annule en raison d'une discrimination (directe) fondée sur l'âge, la décision du Recteur de Paris refusant d'inscrire au concours second concours d'agrégation un candidat ${ }^{24}$. Le Défenseur des droits a décidé de présenter des observations le 25 janvier 2015, dans lesquelles il démontrait de manière parfaitement convaincante que «la légitimité de l'objectif assigné à la condition d'âge par l'administration» était fragile, et «la mesure pour y parvenir, résolument disproportionnée au but recherché ». Les conclusions de Rémi Keller n'ont pas fait mention de ces observations, et pour cause, ces dernières ont été présentées après l'audience. Toutefois, les mêmes observations avaient précédemment été présentées au tribunal administratif de Versailles, incompétemment saisi et la formation de jugement en avait nécessairement eu connaissance. Les conclusions de Remi Keller n'en font pas mention non plus. À en lire pourtant les observations de suivi sur le site du Défenseur des droits, «Le Conseil d'État suit les observations du Défenseur et annule la décision du recteur ». On est volontiers enclin à le penser. Mais on ne peut pas le démontrer. Sur un plan formel, l'expression de satisfaction du Défenseur des droits est impropre, car le juge administratif ne saurait «suivre » un avis du Défenseur des droits, encore moins des observations. Tout n'est qu'influence feutrée. Aucune autre décision du Conseil d'État ne fait référence, en matière de contentieux de la discrimination, aux observations du Défenseur des droits.

Cela est d'autant plus frustrant que, dans la décision Perreux, des délibérations de la HALDE versées ès qualités d'observatrice au dossier, avaient été dûment examinées dans les conclusions du Rapporteur public comme éléments de présomption, mais aussi dûment mentionnées et considérées dans leur vertu probatoire (mais pas en l'espèce) dans le corps même de la décision : "Considérant (...) que ces éléments de fait sont corroborés par une délibération en date du 15 septembre 2008 de la Haute Autorité de lutte contre les discriminations et pour l'égalité, que cette dernière a entendu verser au dossier de la procédure en application (...) de la loi du 30 décembre $2004 »{ }^{25}$. Mais depuis la décision fondatrice, plus aucune mention des observations n'a été observée.

Devant les juges du fond, l'influence est à peine plus assumée. À l'issue des recherches de jurisprudences, on isole trois jugements de tribunaux administratifs s'appuyant

\footnotetext{
${ }^{24}$ CE 26 janvier 2015, Slama, n 373746.

${ }^{25}$ CE, Ass., 30 octobre 2009, Perreux, nº 298348.
} 
formellement sur les observations du Défenseur des droits ${ }^{26}$. Le plus récent mérite l'attention. Le Défenseur des droits a publié sur son site internet des observations présentées devant le tribunal administratif de Dijon sur des faits de harcèlement discriminatoire fondé sur l'origine et le sexe et de harcèlement sexuel à l'encontre d'une femme recrutée dans à la Compagnie républicaine de sécurité ${ }^{27}$. À l'issue de son instruction, le Défenseur des droits estime que seul le harcèlement sexuel est parfaitement établi. La discrimination fondée sur le sexe est également établie et c'est sur ce point que les données empiriques fournies au juge par le Défenseur sont intéressantes puisqu'il va lui-même puiser ses propres données ${ }^{28}$ qui renvoient à d'autres données empiriques, en l'espèce une étude du Conseil supérieur de l'égalité professionnelle entre les femmes et les hommes (CSEP, 2015). Aux termes de celle-ci, un sexisme hostile est ouvertement exprimé «dans deux espaces bien identifiables : les métiers traditionnellement masculins et la gouvernance ». Le jugement du 31 décembre 2018 retient le harcèlement sexuel et la discrimination sexiste en faisant ouvertement référence aux observations du Défenseur des droits s'agissant, notamment de l'«ambiance sexiste », et d' « un contexte intimidant et fortement hostile, ainsi qu'un traitement défavorable des agents de sexe féminin au sein des CRS à raison précisément de leur sexe ». Il est très net dans cette illustration que les données fournies par le Défenseur ont considérablement éclairé l'instruction. Mais cela reste rare. Quant aux décisions des cours administratives d'appel, sur une cinquantaine recensée la plupart ne font pas référence aux observations du Défenseur et se bornent à les viser. D'autres mentionnent et s'appuient sur leur contenu pour valider l'existence d'une discrimination ${ }^{29}$, ou pour ne pas leur accorder de valeur probatoire ${ }^{30}$, ou bien sûr pour écarter le moyen tiré d'une discrimination lorsque le Défenseur lui-même ne l'a pas reconnue ${ }^{31}$. Naturellement, lorsque les recommandations du Défenseur (qui sont différentes des observations présentées en justice) sont versées au dossier, elles deviennent une pièce d'instruction classique et il y est plus facilement fait référence. Même constat pour des décisions prises antérieurement par le Défenseur sur l'objet du litige, qui viennent s'ajouter au faisceau de présomptions, tout en étant intrinsèquement «dépourvues de force contraignante $»^{32}$.

Les observations du Défenseur des droits ont donc, à défaut de statut procédural déterminant, un poids probable sur le processus du juge pour se forger une conviction. Mais la lecture des décisions et des observations correspondantes trouvées, combinées à celle des conclusions du Rapporteur public lorsqu'elles sont disponibles, font assez peu apparaitre ce poids et ne laisse que présumer d'une influence.

\section{B. Des influences diffuses}

Il existe une autre influence encore plus subtile, encore moins saisissable. C'est celle émanant de la littérature «instituée » évoquée plus haut, au sein de laquelle on peut d'ailleurs retrouver des productions du Défenseur autres que des observations en justice (Bretonneau, 2016). Mais l'analyse des rapports d'influence devient difficile. D'abord parce qu'on ne retrouve de références que dans les conclusions du Rapporteur public et que le Rapporteur public n'appartient pas à la formation de jugement et peut n'être pas suivi. Ensuite parce pour s'aventurer dans une telle recherche, il faut un solide fil d'Ariane. Malheureusement, nous

\footnotetext{
${ }^{26}$ TA Cergy-Pontoise, 16 avril 2016, n¹605813; TA Montpellier, 16 février 2018, ${ }^{\circ} 1600328$; TA Dijon, 31 décembre $2018, \mathrm{n}^{\circ} 1702154$.

${ }^{27}$ Décision 2018-105 du 4 juin 2018 relative à des faits de harcèlement discriminatoires fondés sur l'origine et le sexe et de harcèlement sexuel.

${ }^{28}$ Décision MLD-2015-249 du 4 décembre 2015.

${ }^{29}$ CAA Nancy, 5 juin 2018, $\mathrm{n}^{\circ} 16 \mathrm{NC} 02639$ et CAA Bordeaux, 10 mars 2014, $\mathrm{n}^{\circ} 13 \mathrm{BX} 00711$.

${ }^{30}$ CAA Douai, 17 août 2017, $\mathrm{n}^{\circ}$ 15DA00575.

${ }^{31}$ CAA Nantes, 29 octobre 2015, n ${ }^{\circ}$ 14NT01549.

${ }^{32}$ CAA Nantes, ler mars 2016, ${ }^{\circ}$ 15NT02459.
} 
devons nous contenter de la fine toile d'ArianeWeb et des revues spécialisées, ce qui limite l'analyse.

La combinaison de toutes ces difficultés nous offre une seule illustration. En 2010, le Conseil d'État statue sur la transmission d'une question prioritaire de constitutionnalité au sujet d'une loi faisant une distinction au sein des occupants sans titre du domaine public et isolant un traitement particulier pour les «gens du voyage » pour qui «l'habitat traditionnel est constitué de résidences mobiles $»^{33}$. Dans ses conclusions, Jean-Philippe Thiellay (Thiellay, 2010) se penche sur le moyen tiré d'une discrimination ethnique et s'appuie notamment sur la littérature de la $\mathrm{CNCDH}$ pour souligner que la loi ne vise en effet que des "groupes ethniques bien identifiés: les Tsiganes, avec notamment les Manouches ou les Sintis, les Gitans et les Roms ou les Yéniches » s'inscrivant dans une tradition ancestrale, et non pas tous les autres, qui vivent ainsi par choix ou nécessité. Convaincu que «le champ d'application de la loi est clairement "ethnique" », il estime qu'une réserve d'interprétation sur la notion d'habitat traditionnel serait la bienvenue. La décision du Conseil d'État (qui transmettra en effet la QPC) ne relaye aucune de ces interrogations, et le Conseil constitutionnel, dans sa décision du 9 juillet $2010^{34}$, répondra qu'il n'y a pas d'atteinte au principe d'égalité (Aubin, 2010).

En somme, le Conseil d'État n'est pas transparent sur l'utilisation des savoirs extérieurs et des données recueillies par les autres. Au-delà de la décision juridictionnelle, ses voix officielles revendiquent pourtant la pratique, à tout le moins en ce qui concerne le Défenseur des droits. «En dehors de toute intervention de la part du Défenseur des droits, il arrive que les juridictions s'appuient sur ses constatations et sur ses rapports », disait Bernard Stirn (Stirn, 2015). "Nous devons en particulier poursuivre le dialogue entre ordres de juridictions, mais aussi avec toutes les institutions, comme le Défenseur des droits, qui participent à la lutte contre les discriminations », rappelait Bruno Lasserre en précisant que « ce faisant nous nous donnons les moyens de perfectionner nos outils de contrôle et d'affiner nos raisonnements. Nous le devons à nos concitoyens et, notamment, à tous ceux qui souffrent de discrimination. » (Lasserre, 2018)

$$
*
$$

L'intervention, sollicitée ou non, des savoirs extérieurs n'a, pour l'heure, pas engagé le juge administratif sur la voie d'une grille de lecture des mesures de la discrimination. Mais cela n'est pas définitivement exclu, pour plusieurs raisons. En premier lieu grâce à un possible phénomène d'utilisation des données empiriques par ricochet. Lorsque le Défenseur des droits, dont on a vu qu'il était une source validée par le Conseil d'État, transmet au juge des outils de mesures et de compréhension, y compris statistiques et en faisant volontiers appel à des données empiriques non hexagonales, il s'émancipe du tabou français sur l'outil statistique et la mesure de la discrimination et peut ainsi créer une interférence avec le classicisme observé. En deuxième lieu parce que le maniement des discriminations indirectes n'a finalement que dix ans d'expérience et va nécessairement évoluer avec les revendications. Cette évolution sera peut-être d'ailleurs observée à travers les actions de groupes introduites par la loi du 18 novembre 2016 de modernisation de la justice du $\mathrm{XXI}^{\mathrm{e}}$ siècle qui prévoit explicitement la possibilité d'une telle action dans le champ des discriminations au recrutement.

Références bibliographiques :

Aubin, Emmanuel (2010), «L'évacuation forcée des gens du voyage; une décision QPC tombant de Charybde en Scylla », AJDA, n 41, p. 2324-2329.

\footnotetext{
${ }^{33}$ CE 28 mai 2010, n 337840.

${ }^{34}$ Décision du 9 juillet 2010, ${ }^{\circ} 2010-13$ QPC.
} 
Bretonneau, Aurélie (2016), Concl. sur CE, 13 juin 2016, Coulibaly, n 372721, ArianeWeb.

Cafarelli, François (2007), Recherche sur le fondement juridique des discriminations compensatoires en droit public français, Thèse, Montpellier, 604 p.

Calvès, Gwénaële (2009), « Le contrôle juridictionnel des questions posées lors de l'épreuve d'entretien $», \mathrm{n}^{\circ} 25$, p. 1386-1389.

Calvès, Gwénaële (2012), « Non-discrimination et égalité : de la fusion à la séparation ? », in F. Fines, C. Gauthier et M. Gautier, La non-discrimination entre les européens, éd. Pedone.

Conseil supérieur de l'égalité professionnelle entre les femmes et les hommes (2015), Le sexisme dans le monde du travail, entre déni et réalité, La documentation française.

Dumortier, Gaëlle. (2012), «L'effet direct des conventions internationales », RFDA, n³, p. 547-560.

Dumortier, Thomas. (2017), «Les discriminations dans la fonction publique : une réponse juridictionnelle limitée », AJDA, n 12 , p. 661-667

Guyomar, Mathias (2009), Concl. sur CE, Ass., 30 octobre 2009, Perreux, n 298348, Recueil Lebon, p. 407.

Lantero, Caroline (2009), «L'impact de la reconnaissance institutionnelle des minorités sur la discrimination », RDP, p. 817-852.

Lasserre, Bruno (2018), Discours prononcé à l'École nationale de la magistrature le jeudi 29 novembre 2018.

Lieber, Sophie-Justine (2018), «De l'usage des statistiques dans la présomption des discriminations : oui, mais... », AJFP, $\mathrm{n}^{\circ} 1$, p. 35-40.

Liéber, Sophie-Justine et Botteghi, Damien (2009), «Mme Perreux (II) - Juger des discriminations, une mission sous contraintes », AJDA, n43, p. 2391-2396.

Melleray, Fabrice (2014), « La réforme de l'expertise », AJDA, n²4, p. 13641370.

Mercat-Bruns, Marie et al. (2017), Les juridictions et les instances publiques dans la mise en œuvre de la non-discrimination : perspectives pluridisciplinaires et comparées, Ministère de la Justice, Rapport de recherche.

Pellissier, Gilles (1996), Le principe d'égalité en droit public, LGDJ.

Roman, Diane (2017), «Fin des menus de substitution dans les cantines scolaires : "Cochon qui s'en dédit!" », AJDA, n³8, p. 2207-2213.

Roussel, Sophie et Nicolas, Charline, «Discrimination : la preuve par la statistique », AJDA, $\mathrm{n}^{\circ} 38$, p. 2193-2198.

Stirn, Bernard (2015), «Le juge administratif et les discriminations », Intervention lors du Colloque «10 ans de droit de la non-discrimination » organisé par le Défenseur des droits à la Cour de cassation le 5 octobre 2015.

Tharaud, Delphine (2013), Contribution à une théorie générale des discriminations positives, PUAM.

Thiellay, Jean-Philippe (2010), » Renvoi au Conseil constitutionnel de la loi de 2007 sur l'évacuation des gens du voyage », AJDA, n²4, p. 1376-1380. 